\title{
MEROMORPHIC FUNCTIONS WITH SIMULTANEOUS MULTIPLICATION AND ADDITION THEOREMS
}

BY

\section{IRVING GERST}

Introduction. Let $f(z)$ be a nonconstant meromorphic function. Further, let there exist two numbers $m \neq 0,1$ and $h \neq 0$, and two rational functions $R$ and $S$, such that

$$
f(m z)=R[f(z)],
$$

and

$$
f(z+h)=S[f(z)] .
$$

Then the function $f(z)$ has both a rational multiplication and a rational addition theorem. We propose to determine all such functions.

Our problem is a generalization of one treated in a paper by $\operatorname{Ritt}\left({ }^{1}\right)$, in which all periodic meromorphic functions having a multiplication theorem were determined. This is equivalent to taking $S(z) \equiv z$ in (2). For this case, Ritt proved that $|m| \geqq 1$; and if $|m|>1$, then $f(z)$ is a linear function of one of the functions $e^{\alpha z}, \cos (\alpha z+\beta), \varphi(z+\beta)$; when $g_{3}=0, \varphi^{2}(z+\beta)$; when $g_{2}=0$, $\gamma^{\prime}(z+\beta)$ and $\varepsilon^{3}(z+\beta)$. Here $\alpha$ is arbitrary while $\beta$ is restricted to certain values. If $|m|=1$, then it was shown that $m$ is either -1 or a third, fourth or sixth root of unity. The forms for the function $f(z)$ were also explicitly given in this case. Use will be made of the results quoted and also of the methods of Ritt's paper.

We distinguish the following three cases:

(A) $|m|>1$;

(B) $|m| \leqq 1$, with $m$ not a rational root of unity;

(C) $m$ a primitive $n$th root of unity.

For Case (A), which is the one usually considered in multiplication theorems, we can restate the problem in a way which shows its connection with the theory of a class of functions first systematically studied by Poincaré( $\left.{ }^{2}\right)$. He provided an existence theorem for meromorphic functions satisfying (1) assuming that $R(z)$ has a fixed point $\left({ }^{3}\right) a$ for which $R^{\prime}(a)$ has a modulus greater than unity. Thus our problem is equivalent to that of finding all Poincare functions having a rational addition theorem.

Presented to the Society, February 22, 1947; received by the editors June 19, 1946.

(1) Ritt, Periodic functions with a multiplication theorem, Trans. Amer. Math. Soc. vol. 23 (1922) pp. 16-25.

(2) H. Poincaré, Sur une classe nouvelle de transcendantes uniformes, Journal de Mathematiques (3) vol. 55 (1890).

(3) That is, a point for which $R(a)=a$. 
We remark that, if $f(z)$ is a linear function of $z$, integral or fractional, then $f(z)$ will evidently have both a multiplication and addition theorem for every value of $m$ and $h$. This solution is therefore common to all three cases and, as it turns out, is the only rational solution of our problem.

On the other hand, if $f(z)$ is transcendental, we may state the chief result of this paper for Case (A) as follows:

If a Poincare function has a rational addition theorem, it must be a periodic function.

Although $f(z)$ is periodic in this case, the value of $h$ in the addition theorem is not necessarily a period, and therefore the corresponding $S(z)$ need not equal z. The possibilities for $h$ and $S(z)$ are given in detail in $\S 4$. It is found that except for the case in which $f(z)$ is a linear function of $e^{\alpha z}, h$, when not a period, must be a suitable half or third of a period. It is noteworthy that for each of these admissible values of $h, S(z)$ is a linear function of $z$.

In Case (B), $f(z)$ must be a linear function of $z$.

In Case (C) there appear nonperiodic solutions in addition to the types already mentioned.

It will be noted that, essentially, only the conditions that $f(m z)$ and $f(z+h)$ are uniform functions of $f(z)$ will enter into consideration.

1. Preliminary transformations. The case of $f(z)$ rational will be handled in $\S 5$. From this point on through $\S 4$, we assume that $f(z)$ is transcendental.

Supposing that $|m|>1$, we wish to replace $f(z)$. by another meromorphic function $g(z)$, related to it in a simple way, but having the following special properties at the origin:

( $\alpha) g(0)=0$;

(ß) $g^{\prime}(0) \neq 0$;

$(\gamma) 0$ is not an exceptional point of $g(z)$; that is, $g(z)$ has an infinite number of zeros.

We further require that there exist relations of the form

$$
g(M z)=U[g(z)],
$$

and

$$
g(z+h)=V[g(z)] .
$$

Here $U$ and $V$ are rational and $M$ is a number for which $|M|>1$.

To find such a function $g(z)$, we consider $g(z) \equiv f(z+\xi)-f(\xi)$, where $f(z)$ is analytic at $\xi$. We shall show that $\xi$ can be chosen so that the resulting function $g(z)$ fulfills all the conditions placed upon it.

Evidently for any value of $\xi$ at which $f(z)$ is analytic, property $(\alpha)$ holds, and also a relation of type (4) exists with $V(z) \equiv S[z+f(\xi)]-f(\xi)$.

Write $\sigma(z) \equiv m z, \tau(z) \equiv z+h$. Let a positive integral subscript appended to a function denote the corresponding iterate of that function; for example $R_{r}(z)$ denotes the $r$ th iterate of $R(z)$. Then if $\xi$ is a finite fixed point of one of 
the transformations $\sigma$ or $\tau \sigma_{r}(r=1,2, \cdots)$ and if $f(z)$ is analytic at $\xi$, a relation of type (3) will exist.

For if $\xi=0$, the finite fixed point of $\sigma$, we may take $M=m$ and $U(z)$ $=R[z+f(0)]-f(0)$.

And if for a positive integral $r, \tau \sigma_{r}(\xi)=\xi$, we may take $M=m^{r}$ and $U(z)=S R_{r}[z+f(\xi)]-f(\xi)$. This follows since

$$
g(M z)=g\left[\sigma_{r}(z)\right]=f\left[\sigma_{r}(z)+\xi\right]-f(\xi)=f\left[\tau \sigma_{r}(z+\xi)\right]-f(\xi) ;
$$

and using (1) and (2) we have

$$
f\left[\tau \sigma_{r}(z+\xi)\right]=S,\left\{f\left[\sigma_{r}(z+\xi)\right]\right\}=S R_{r}[f(z+\xi)] .
$$

In each of these cases $|M|>1$.

It remains to show the existence of a fixed point $\xi$ at which $f(z)$ is analytic and for which the corresponding function $g(z)$ has properties $(\beta)$ and $(\gamma)$. To this end consider the sequence

$$
0, \frac{h}{1-m}, \frac{h}{1-m^{2}}, \cdots, \frac{h}{1-m^{r}}, \cdots,
$$

consisting of the finite fixed points of $\sigma$ and $\tau \sigma_{r}(r=1,2, \cdots)$.

These points converge to the origin since $|m|>1$. Hence there exists a point $\xi$ in the above sequence at which $f(z)$ is analytic, at which $f^{\prime}(z)$ is not zero, and which is not an exceptional point of $f(z)$ in the sense of Picard. With this value of $\xi$, the corresponding function $g(z)$ will have the required properties $(\beta)$ and $(\gamma)$.

2. The automorphism of $g(z)$. Adapting a method of Ritt $\left({ }^{4}\right)$, we now establish a relation for $g(z)$ which will enable us to show that $g(z)$ is periodic.

As $g(z)$ has an infinite number of zeros, suppose that $z_{1} \neq 0$ is one of them. Then, since $g(0)=0$ and $g^{\prime}(0) \neq 0$, there exists a neighborhood of the origin, $\Gamma_{0}$, in which $g(z)$ assumes no value more than once.

Since $g\left(z_{1}\right)=0$, there exists a neighborhood $\Gamma_{1}$ of $z_{1}$, in which $g(z)$ takes no value not assumed in $\Gamma_{0}$.

Define $\phi(z)$, for $z$ in $\Gamma_{1}$, as that unique point $z_{2}$ of $\Gamma_{0}$ for which $g\left(z_{2}\right)=g(z)$. Then

$$
g[\phi(z)] \equiv g(z)
$$

for $z$ in $\Gamma_{1}$.

The function $\phi(z)$ is analytic in $\Gamma_{1}$, not identically 0 , and has a Taylor development at $z_{1}$. Since $\phi\left(z_{1}\right)=0$, we may write that expansion as

$$
\phi(z)=\alpha_{1}\left(z-z_{1}\right)+\alpha_{2}\left(z-z_{1}\right)^{2}+\cdots+\alpha_{n}\left(z-z_{1}\right)^{n}+\cdots .
$$

(4) Ritt, loc. cit. pp. 17-19. 
It will now be shown that $\phi(z)$ is linear. Then evidently $\alpha_{1} \neq 0$, since $\phi(z) \not \equiv 0$ and our argument will show that $\alpha_{n}=0$ for $n>1$. Take $s$, an integer, so great that $z_{1}+h / M^{*}$ is in $\Gamma_{1}$. Then, from (5),

$$
g\left[\phi\left(z_{1}+\frac{h}{M^{*}}\right)\right]=g\left(z_{1}+\frac{h}{M^{s}}\right) .
$$

Now by (3) and (7) with $U_{s}$ denoting the $s$ th iterate of $U$, we have

$$
g\left[M^{s} \phi\left(z_{1}+\frac{h}{M^{s}}\right)\right]=U_{s}\left\{g\left[\phi\left(z_{1}+\frac{h}{M^{s}}\right)\right]\right\}=U_{s}\left[g\left(z_{1}+\frac{h}{M^{s}}\right)\right],
$$

or

$$
g\left[M^{*} \phi\left(z_{1}+\frac{h}{M^{*}}\right)\right]=g\left[M^{*}\left(z_{1}+\frac{h}{M^{*}}\right)\right]=g\left[M^{s} z_{1}+h\right] .
$$

Using (4) and then (3), we find

$$
g\left[M^{\prime} z_{1}+h\right]=V\left[g\left(M^{s} z_{1}\right)\right]=V U_{s}\left[g\left(z_{1}\right)\right] .
$$

But, since $g\left(z_{1}\right)=0, V U_{\bullet}\left[g\left(z_{1}\right)\right]=V U_{\imath}(0)=V(0)$. Thus (8) becomes

$$
g\left[M^{*} \phi\left(z_{1}+\frac{h}{M^{*}}\right)\right]=V(0)
$$

We have by (6)

$$
M^{\circ} \phi\left(z_{1}+\frac{h}{M^{s}}\right)=\alpha_{1} h+\alpha_{2} \frac{h^{2}}{M^{s}}+\cdots .
$$

Then by (9)

$$
g\left(\alpha_{1} h+\alpha_{2} \frac{h^{2}}{M^{2}}+\cdots\right)=V(0)
$$

for sufficiently large $s$.

It follows from (10) that $\alpha_{n}=0$ for $n>1$, otherwise allowing $s$ to pass through all sufficiently large values we would have an infinite number of distinct points accumulating at the point $\alpha_{1} h$ for each of which $g(z)$ assumes the value $V(0)$, and $\alpha_{1} h$ would be an essential singularity of $g(z)$.

Thus (5) becomes

$$
g\left[\alpha_{1}\left(z-z_{1}\right)\right] \equiv g(z) .
$$

This equation, proved for $z$ in $\Gamma_{1}$, must hold for the whole plane since the functions in it are analytic.

This is the desired relation.

3. Proof of periodicity. It now follows that $g(z)$ is periodic. For in (11), 
if $\alpha_{1}=1,-z_{1}$ is a period of $g(z)$. If $\alpha_{1} \neq 1$, then by using (4) and (11) we find

$$
g(z+h)=V\left\{g\left[\alpha_{1}\left(z-z_{1}\right)\right]\right\}=g\left[\alpha_{1}\left(z-z_{1}\right)+h\right] .
$$

But from (11) with $z$ replaced by $z+h$, we get

$$
g(z+h)=g\left[\alpha_{1}\left(z-z_{1}\right)+\alpha_{1} h\right] .
$$

Then

$$
g\left[\alpha_{1}\left(z-z_{1}\right)+h\right]=g\left[\alpha_{1}\left(z-z_{1}\right)+\alpha_{1} h\right],
$$

and $h\left(\alpha_{1}-1\right) \neq 0$ is a period of $g(z)$.

The function $f(z)$ which is $g(z)+f(0)$ or $g\left[z-h /\left(1-m^{r}\right)\right]+f\left[h /\left(1-m^{r}\right)\right]$ is, of course, also periodic. This is the result stated in the introduction.

4. The values of $h$. Having established the periodicity of our functions, our next step is to determine the values of $h$ for which addition theorems are possible if a multiplication theorem also holds. Evidently $h$ may be a period and then $S(z) \equiv z$. But there are other values besides periods and it is these in which we are particularly interested.

Use will be made of the fact that together with $f(z), F(z)=[A f(z)$ $+B] /[C f(z)+D]$ will also have a multiplication and addition theorem. In what follows, we will use $F(z)$ to denote such a linearly transformed $f(z)$.

As stated in the introduction, the periodic functions with multiplication theorems are divided into six categories and each of these will be considered in turn.

Case (a): $f(z)$ a linear function of $e^{\alpha z}$. Here $\alpha$ is arbitrary and the multiplier $m$ must be an integer.

Replacing $f(z)$ by a suitable linear function of itself, we may suppose that

$$
F(z)=e^{\alpha z} \text {. }
$$

Then since

$$
F(z+h)=e^{\alpha h} F(z),
$$

$F(z)$ and therefore $f(z)$ has an addition theorem for every value of $h$.

Case (b): $f(z)$ a linear function of $\cos (\alpha z+\beta)$. Here $\beta=k \pi /(m-1), k$ an integer, while $\alpha$ is arbitrary. The multiplier $m$ must be an integer.

We may suppose

$$
F(z)=\cos (\alpha z+\beta),
$$

and have to determine for which values of $h, \cos (\alpha z+\alpha h+\beta)$ is a rational function of $\cos (\alpha z+\beta)$. Replacing $\alpha z+\beta$ by $z$ and $\alpha h$ by $\gamma$, we must have

$$
\cos (z+\gamma)=S[\cos z] \text {, }
$$

with $S$ rational.

Let $z_{1}$ be any value of $z$ and determine another value of $z, z_{2}$ so as to satisfy the congruence 


$$
z_{1}+z_{2} \equiv 0(\bmod 2 \pi)
$$

Then, since $\cos z_{1}=\cos z_{2}$,

$$
\cos \left(z_{1}+\gamma\right)=\cos \left(z_{2}+\gamma\right) .
$$

We have the following possibilities; either

$$
z_{1}+\gamma \equiv z_{2}+\gamma(\bmod 2 \pi),
$$

or

$$
z_{1}+\gamma+z_{2}+\gamma \equiv 0(\bmod 2 \pi),
$$

or perhaps both of these congruences hold.

In any event, if (13) holds, then adding (12) to it we get

$$
2 z_{1} \equiv 0(\bmod 2 \pi) \text {; }
$$

that is, $z_{1}=k \pi, k$ an integer. If $z_{1}$, which was arbitrary, is given any value not of the form $k \pi$, only $\left(13^{\prime}\right)$ can hold. We may suppose this done. Then the subtraction of (12) from $\left(13^{\prime}\right)$ gives

$$
2 \gamma \equiv 0(\bmod 2 \pi)
$$

that is, $\gamma=k \pi, k$ an integer. Then $h=k \pi / \alpha$.

Since $2 \pi / \alpha$ is a period of $\cos (\alpha z+\beta)$, the values of $h$, other than the periods, must be the half-periods.

That this necessary condition for $h$ is also sufficient is obvious. Thus, for $h$ the half-period $\pi / \alpha$, to which all other half-periods are congruent modulo $2 \pi / \alpha$, we have

$$
\cos [\alpha(z+\pi / \alpha)+\beta]=-\cos (\alpha z+\beta) .
$$

Case (c): $f(z)$ a linear function of $p(z+\beta)$. The multiplier $m$ must satisfy the congruences

$$
2 m \omega_{1} \equiv 0, \quad 2 m \omega_{3} \equiv 0\left(\bmod 2 \omega_{1}, 2 \omega_{3}\right),
$$

and the constant $\beta$ is given by the equation

$$
\beta=\frac{l \omega_{1}+k \omega_{3}}{m-1},
$$

where $l$ and $k$ are integers, and $2 \omega_{1}, 2 \omega_{3}$, a pair of primitive periods of $8(z)$.

By repeating the procedure of the preceding case, except that all congruences are taken modulis $2 \omega_{1}, 2 \omega_{2}$ we find that

$$
h=l \omega_{1}+k \omega_{3} \text {, }
$$

where $l$ and $k$ are any integers.

Again we get periods and half-periods for $h$. 
To set up the addition theorem for the half-periods, it suffices to consider $\omega_{1}, \omega_{3}$ and $\omega_{2}=-\omega_{1}-\omega_{3}$, for any other half-period will be congruent to one of these modulis $2 \omega_{1}, 2 \omega_{3}$.

The following formula is well known in the theory of elliptic functions ( 5$)$

$$
\varphi\left(z+\omega_{a}\right)=e_{a}+\frac{\left(e_{b}-e_{a}\right)\left(e_{c}-e_{a}\right)}{\varphi(z)-e_{a}},
$$

where $\varphi\left(\omega_{a}\right)=e_{a}$ and $a, b, c$ is any permutation of $1,2,3$.

This is the required addition theorem if $z$ is replaced by $z+\beta$.

Case $(\mathrm{d}): f(z)$ a linear function of $q^{2}(z+\beta)$. Here $q(z)$ is lemniscatic, $m=p+q i$, with $p$ and $q$ integral, and

$$
\beta=\frac{2 l \omega_{1}+2 k \omega_{3}}{(m-1)(1-i)},
$$

where $l$ and $k$ are any integers.

We may replace $z+\beta$ by $z$ and we shall suppose this done here and in the following cases. The values of $h$ do not depend on those of $\beta$.

Taking $F(z)=q^{2}(z)$, we must have $q^{2}(z+h)=S\left[\wp^{2}(z)\right]$.

Since $\vartheta^{2}(i u)=\wp^{2}(u)$ in this case, the fourth order function $\varphi^{2}(u)$ will take on the same value at points congruent to $i^{s} u(s=0,1,2,3)$ modulis $2 \omega_{1}, 2 \omega_{3}$, and only at these points if the $i^{s} u$ are all incongruent.

If any two of $i^{i} u$ are congruent, it follows that they are all congruent, and $8^{2}(u)$ will have a point of the fourth order at any one of them; so that again $\gamma^{2}(u)$ will take the same value only at the points $i^{s} u$.

Choose an arbitrary $z_{1}$, and determine $z_{2}$ so as to satisfy the congruence

$$
z_{2} \equiv i z_{1}\left(\bmod 2 \omega_{1}, 2 \omega_{3}\right) \text {. }
$$

Then since $\varphi^{2}\left(z_{2}\right)=\varphi^{2}\left(z_{1}\right)$, it follows that

$$
\boldsymbol{p}^{2}\left(z_{2}+h\right)=\boldsymbol{p}^{2}\left(z_{1}+h\right),
$$

and

$$
z_{2}+h \equiv i^{\bullet}\left(z_{1}+h\right)\left(\bmod 2 \omega_{1}, 2 \omega_{8}\right),
$$

where $s$ may be one of $0,1,2,3$, or perhaps any of them.

Subtracting (15) from this congruence gives

$$
h\left(1-i^{s}\right) \equiv\left(i^{s}-i\right) z_{1}\left(\bmod 2 \omega_{1}, 2 \omega_{3}\right) ;
$$

and unless $s=1$, this will determine $z_{1}$ as belonging to a particular residue class modulis $2 \omega_{1} /\left(i^{3}-i\right), 2 \omega_{3} /\left(i^{3}-i\right)$, whereas $z_{1}$ was chosen without restriction.

If $s=1$, we find that

(5) Tannery and Molk, Théorie des functions élliptiques, vol. 1, p. 193. 


$$
h=\frac{2 l \omega_{1}+2 k \omega_{8}}{1-i}
$$

where $l$ and $k$ are any integers.

Simplifying this expression, we get $h=(l-k) \omega_{1}+(l+k) \omega_{3}$. Thus the values of $h$ must be periods or suitable half-periods; namely, since $l-k$ and $l+k$ are both of the same parity, those half-periods which are congruent to $\omega_{2}$ modulis $2 \omega_{1}, 2 \omega_{8}$.

If we note that

$$
i \omega_{2} \equiv i\left(-\omega_{1}-\omega_{8}\right) \equiv-\omega_{3}+\omega_{1} \equiv \omega_{2}\left(\bmod 2 \omega_{1}, 2 \omega_{3}\right),
$$

and that $\varphi(i u)=-\varphi(u)$ for this case, then

$$
e_{2}=p\left(\omega_{2}\right)=p\left(i \omega_{2}\right)=-\phi\left(\omega_{2}\right)=-e_{2},
$$

and $e_{2}=0$.

Substituting $a=2$ in (14) and squaring we get the addition formula

$$
\vartheta^{2}\left(z+\omega_{2}\right)=\frac{e_{1}^{2} e_{3}^{2}}{\nabla^{2}(z)} .
$$

Case $(\mathrm{e}): f(z)$ a linear function of $q^{\prime}(z+\beta)$. Here $q^{\prime}(z)$ is equianharmonic, $m=p+q e^{2 \pi i / 3}$, with $p$ and $q$ integral and

$$
(m-1)\left(1-e^{2 \pi i / 3}\right) \beta \equiv 0\left(\bmod 2 \omega_{1}, 2 \omega_{3}\right) .
$$

This case and the next one may be treated in the same way as case (d) and we simply state the results.

Since $\phi^{\prime}\left(e^{2 \pi i / 3} u\right)=\varphi^{\prime}(u)$ in this case, $e^{2 \pi i / 3}$ takes the place of $i$ in the preceding case and thus

$$
h=\frac{2 l \omega_{1}+2 k \omega_{3}}{1-e^{2 \pi i / 8}},
$$

where $l$ and $k$ are any integers.

Rationalizing the denominator of this fraction, we get

$$
h=\frac{2 \omega_{1}(2 l-k)+2 \omega_{3}(l+k)}{3} .
$$

Thus, apart from a period, $h$ must be a suitable third of a period.

To get our addition theorem in the latter instance, we note that taken modulis $2 \omega_{1}, 2 \omega_{3}$ all these thirds of periods are congruent to $\gamma=\left(2 \omega_{1}-2 \omega_{3}\right) / 3$ or $-\gamma$. It suffices to consider $\gamma$. Since $\wp\left(e^{2 \pi i / 3} u\right)=e^{2 \pi i / 3} \&(u)$ and

$$
e^{2 \pi i / 3} \gamma \equiv \gamma\left(\bmod 2 \omega_{1}, 2 \omega_{3}\right),
$$

we have 


$$
\ell(\gamma)=\varnothing\left(e^{2 \pi i / 3} \gamma\right)=e^{2 \pi i / 3} \varphi(\gamma)
$$

and $\varphi(\gamma)=0$.

If we let $u=\gamma$ in the addition formula for $\varphi(z+u)\left({ }^{6}\right)$ and differentiate the resulting expression with respect to $z$, we find

$$
\phi^{\prime}(z+\gamma)=\frac{\phi^{\prime}(z) \phi^{\prime}(\gamma)-3\left[\gamma^{\prime}(\gamma)\right]^{2}}{\wp^{\prime}(z)+\phi^{\prime}(\gamma)} .
$$

Case $(f): f(z)$ a linear function of $\varphi^{3}(z+\beta)$. Here $\varphi(z)$ is equianharmonic, the multiplier $m=p+q e^{2 \pi i / 3}$, with $p$ and $q$ integral, and

$$
(m-1)\left(1-e^{\pi i / 3}\right) \beta \equiv 0\left(\bmod 2 \omega_{1}, 2 \omega_{3}\right) .
$$

Since $\varphi^{3}\left(e^{\pi i / 3} u\right)=\wp^{3}(u)$, we may use $e^{\pi i / 3}$ in the same manner as $e^{2 \pi i / 3}$ and $i$ in the preceding two cases. The result is that

$$
h=\frac{2 l \omega_{1}+2 k \omega_{3}}{1-e^{\pi i / 8}},
$$

with $l$ and $k$ any integers.

This simplifies to

$$
h=\left(1+e^{2 \pi i / 3}\right)\left(2 l \omega_{1}+2 k \omega_{3}\right) .
$$

Then the only values of $h$ in this case are periods of $f(z)$.

5. The case of $|m| \leqq 1, m$ not a rational root of unity. We first show that, in this case, $f(z)$ must be rational. For otherwise, suppose that $f(z)$ is transcendental.

Then again considering the sequence

$$
0, \frac{h}{1-m}, \frac{h}{1-m^{2}}, \cdots, \frac{h}{1-m^{r}}, \cdots,
$$

whose points are all distinct, we can find a point in it which is not an exceptional point of $f(z)$. If $f(z)$ is analytic at this point, the transformations of $\$ 1$ will then give us a $g(z)$ having properties $(\alpha)$ and $(\gamma)$ and satisfying relations of type (3) and (4).

If $f(z)$ has a pole at the non-exceptional point, we take $g(z)=1 / f(z)$ or $g(z)=1 / f\left[z+h /\left(1-m^{r}\right)\right]$ according as our point is 0 or $h /\left(1-m^{r}\right)$, and again get a $g(z)$ fulfilling these same four conditions. In either case, $|M| \leqq 1, M$ not a rational root of unity.

If $z_{1} \neq 0$ is one of the zeros of $g(z)$, then the relation

$$
g(M z)=U[g(z)]
$$

shows that $M^{*} z_{1}$ is a zero of $g(z)$ for every positive integral $s$. If $|M|<1$, these

(') See, for example, Tannery and Molk, loc. cit. p. 172. 
zeros will converge to the origin; if $|M|=1, M$ not a rational root of unity, they will be everywhere dense on the circle $|z|=\left|z_{1}\right|$. Both of these results are impossible for a nonconstant meromorphic function.

Therefore, let $f(z)$ be rational $\left({ }^{7}\right)$. Then $R$ and $S$ are both linear. Replacing $f(z)$ by a suitable linear function of itself, which will also be rational, we may obtain the addition theorem in one of the forms:

$$
F(z+h)=a F(z),
$$$$
a \neq 1
$$

or

$$
F(z+h)=F(z)+b .
$$

The first of these is impossible for a nonconstant rational $F(z)$, since the existence of a zero or a pole at $z_{1}$ would imply the existence of an infinite number of zeros or poles at the points $z_{1}+n h, n$ any integer.

The second gives

$$
F(z)=\frac{b z}{h}+\Pi(z),
$$

where $\Pi(z)$ is a meromorphic periodic function of period $h$.

Here $\Pi(z)$ must be rational and therefore constant. Then $F(z)$ and also $f(z)$ is a linear function of $z$.

6. The case $m$ a primitive $n$th root of unity. If $m$ is a primitive $n$th root of unity,

$$
f\left(m^{n} z\right)=f(z)=R_{n}[f(z)],
$$

and the rational function $R(z)$ must be a periodic linear function of $z$ with period $n$ or a divisor of $n$.

For every positive integral $p$ and $r$, we have

$$
f\left(m^{r} z+p h\right)=S_{p}\left[f\left(m^{r} z\right)\right]=S_{p} R_{r}[f(z)] .
$$

Then, if $r$ is not a multiple of $n$, the function $g(z)=f\left(z+p h /\left(1-m^{r}\right)\right)$ obeys the relation

$$
g\left(m^{r} z\right)=S_{p} R_{r}[g(z)] .
$$

This shows that $S_{p} R_{r}(\dot{z})$ is a linear periodic function of $z$ with period $n$ or a divisor of $n$ for every positive integral $p$ and $r, r$ not a multiple of $n$.

The function $S(z)$ itself then must be linear.

Those cases with $R(z)$ of period $d<n$ are easily handled. For with $r=d$ and $p=1, S_{p} R_{r}(z)$ becomes $S(z)$ which must be periodic. Then $f(z)$ is peri$\operatorname{odic}\left({ }^{8}\right)$.

( ${ }^{7}$ ) The following argument holds for any value of $m$. We thus complete our discussion of Case (A) for $f(z)$ rational.

(8) We refer to the paper of Ritt, loc. cit., for the enumeration of the functions in this case. 
In a similar manner, it may be shown that $f(z)$ is periodic if the period of $S_{p} R(z)$ is less than $n$.

These cases disposed of, we may assume both $R(z)$ and $S_{p} R(z)$ to be of period $n(p=1,2, \cdots)$.

Then, replacing $f(z)$ by a suitable linear function of itself, we have two sub-cases according as

$$
F(z+h)=a F(z), \quad a \neq 1
$$

or

$$
F(z+h)=F(z)+b .
$$

Subcase 1: $S(z) \equiv a z, a \neq 1$. Suppose first that $m=-1$, that is, $n=2$. Then $R(z)$, being of period 2 , is of the form $(A z+B) /(C z-A)$. The function $S R(z)=a(A z+B) /(C z-A)$ must also be of period 2 .

If $A \neq 0, a$ must be -1 and $F(z)$ is periodic.

If $A=0, a$ is arbitrary. Solving the addition relation for $F(z)$ by elementary means, we get

$$
F(z)=e^{\alpha z} \Pi(z),
$$

where $\alpha=\log a / h$ and $\Pi(z)$ is a meromorphic function with period $h$.

In order for this function, $F(z)$, to satisfy the multiplication theorem, we find that $\Pi(z)$, a periodic function, must also have a multiplication theorem, namely

$$
\Pi(-z)=\frac{B}{C \Pi(z)} .
$$

Thus $\Pi(z)$ and also $F(z)$ are characterized.

Let us now consider the values of $m$ for which $n>2$. Then with $R(z)$ $=(A z+B) /(C z+D)$, we have

$$
S_{p} R(z)=a^{p} \cdot \frac{A z+B}{C z+D} ;
$$

and this function must be of period $n$ for every positive integral $p$.

The multiplier, $K$, of the periodic linear transformation $S_{p} R(z)$ is a primitive $n$th root of unity which we denote by $\epsilon_{p}$, the subscript indicating its dependence on $p$. Using the known relation between the multiplier and the coefficients of the transformation $\left({ }^{9}\right)$, we find that $K$ is algebraic in $a^{p}$, and we may write

$$
\epsilon_{p}=W\left(a^{p}\right) \quad(p=1,2, \cdots)
$$

where $W(z)$ is an algebraic function of $z$.

(') See, for example, Ford, Automorphic functions, p. 16. 
There being only a finite number of primitive $n$th roots of unity, at least one of them will occur in (16) as $\epsilon_{p}$ an infinite number of times as $p$ grows large. This shows that $a$ must be a rational root of unity since otherwise the algebraic function $W(z)$ would take the same value, $\boldsymbol{\epsilon}_{p}$, at an infinite number of distinct points $z=a^{p}$. Thus $S(z)$ is periodic. We conclude that if $n>2, f(z)$ must be periodic.

Subcase 2: $S(z) \equiv z+b$. If $b=0, f(z)$ is periodic. Suppose $b \neq 0$. Taking $R(z)=(A z+B) /(C z+D)$ with $A D-B C=1$, the linear transformation $S_{p} R(z)$ $=[(A+p b C) z+(B+p b D)] /(C z+D), p=1,2, \cdots$, is periodic and hence elliptic. Using the classic condition that a linear transformation of determinant unity be elliptic $\left({ }^{10}\right)$, we would have $|A+p b C+D|<2$ for every positive integral $p$ and this implies that $C=0$. Then $A=\epsilon D$ where $\epsilon$ is a primitive $n$th root of unity. By taking a suitable linear function of $F(z)$ we may suppose our addition and multiplication theorem in the form

$$
\begin{aligned}
F(m z) & =\epsilon F(z), \\
F(z+h) & =F(z)+b .
\end{aligned}
$$

These show that $F^{\prime}(z)$ is a periodic function with a multiplication theorem. If $F^{\prime}(z)$ is constant, $F(z)$ is a linear function of $z$. If $F^{\prime}(z)$ is not constant; then, as stated in the introduction, $m$ is either -1 or a primitive third, fourth, or sixth root of unity.

Taking the solution of (18) as

$$
F(z)=b z / h+\Pi(z),
$$

where $\Pi(z)$ is a meromorphic function of period $h$, we must determine $\Pi(z)$ so that (17) is also satisfied.

If $m=-1, \Pi(z)$ must be an odd function. Then, if $\Pi(z)$ is simply periodic, it is the product of $\sin (2 \pi n z / h)$ by a meromorphic function of $\cos (2 \pi n z / h)$, $n$ any integer. If $\Pi(z)$ is elliptic, it is the product of $p^{\prime}(z)$ by a rational function of $\boldsymbol{p}(\boldsymbol{z})$.

If $m$ is a primitive fourth root of unity, we may suppose that $m=i$. Then if $\epsilon=i$,

$$
\Pi(i z)=i \Pi(z),
$$

and $\Pi(z)$ is the product of $q^{\prime}(z)$ by a rational function of $p^{2}(z)$ with $q(z)$ lemniscatic.

If $c=-i$, we proceed in a different manner. Let $\zeta(z)$ be the Weierstrass zeta-function corresponding to a lemniscatic $\rho(z)$ having $h$ as a period.

The following relations hold for $\zeta(z)$.

$$
\zeta(i z)=-i \zeta(z), \quad \zeta(z+h)=\zeta(z)+\kappa_{1}, \quad \zeta(z+i h)=\zeta(z)+\kappa_{2} .
$$

Here $\kappa_{1}$ and $\kappa_{2}$ are constants each different from zero. To show this, we have

$\left({ }^{10}\right)$ Ford, loc. cit. p. 23. 
$\zeta(i z)+\kappa_{2}=\zeta(i z+i h)=-i \zeta(z+h)=-i \zeta(z)-i \kappa_{1}=\zeta(i z)-i \kappa_{1}$.

Thus $\kappa_{2}=-i \kappa_{1}$, and the vanishing of either would imply the same for the other. But then $\zeta(z)$ would be elliptic, a contradiction.

If we let

$$
F(z)=b \zeta(z) / \kappa_{1}+\Pi(z),
$$

then (17) and (18) will be satisfied only if $\Pi(z)$ is of period $h$ and obeys the relation

$$
\Pi(i z)=-i \Pi(z) .
$$

Then $\Pi(z)$ is a product of $q^{\prime \prime \prime}(z)$ by a rational function of $q^{2}(z)$ with $\&(z)$ lemniscatic.

For the remaining cases the results are similar.

If $m$ is a primitive cube root of unity,

$$
F(z)=b z / h+\varphi(z) T\left[\wp^{\prime}(z)\right]
$$

or

$$
F(z)=b \zeta(z) / \kappa_{1}+q^{\prime \prime}(z) T\left[\wp^{\prime}(z)\right],
$$

where $\varphi(z)$ is equianharmonic and $T(z)$ is a rational function. If $m$ is a primitive sixth root of unity, we replace $\varphi(z), \varphi^{\prime \prime}(z)$ and $\varnothing^{\prime}(z)$ respectively by $q^{\prime \prime \prime}(z), \rho^{\mathrm{V}}(z)$, and $\varphi^{3}(z)$. In each case it is understood that any linear function of $F(z)$ also has both a multiplication and addition theorem.

Columbia University, NEW YORK, N. Y. 